\title{
Interplay between single-particle and collective excitations in argon isotopes populated by transfer reactions
}

S. Szilner, ${ }^{1}$ L. Corradi, ${ }^{2}$ F. Haas, ${ }^{3}$ D. Lebhertz, ${ }^{3}$ G. Pollarolo, ${ }^{4}$ C. A. Ur, ${ }^{5}$ L. Angus, ${ }^{6}$ S. Beghini,,${ }^{5}$ M. Bouhelal, ${ }^{3}$ R. Chapman, ${ }^{6}$ E. Caurier, ${ }^{3}$ S. Courtin, ${ }^{3}$ E. Farnea, ${ }^{5}$ E. Fioretto, ${ }^{2}$ A. Gadea, ${ }^{2,7}$ A. Goasduff, ${ }^{3}$ D. Jelavić-Malenica, ${ }^{1}$ V. Kumar, ${ }^{6}$ S. Lunardi, ${ }^{5}$ N. Mărginean,,${ }^{2,8}$ P. Mason, ${ }^{5}$ D. Mengoni, ${ }^{5,6}$ G. Montagnoli, ${ }^{5}$ F. Nowacki, ${ }^{3}$ F. Recchia,${ }^{5}$ E. Sahin, ${ }^{2}$ M.-D. Salsac,${ }^{3,9}$

F. Scarlassara, ${ }^{5}$ R. Silvestri, ${ }^{2}$ J. F. Smith, ${ }^{6}$ N. Soić, ${ }^{1}$ A. M. Stefanini, ${ }^{2}$ and J. J. Valiente-Dobón ${ }^{2}$

${ }^{1}$ Ruđer Bošković Institute, HR-10002 Zagreb, Croatia

${ }^{2}$ INFN - Laboratori Nazionali di Legnaro, I-35020 Legnaro, Italy

${ }^{3}$ IPHC, CNRS/IN2P3 and Université de Strasbourg, F-67037 Strasbourg Cedex 2, France

${ }^{4}$ INFN and Università di Torino, I-10125 Torino, Italy

${ }^{5}$ INFN and Università di Padova, I-67037 Padova, Italy

${ }^{6}$ School of Engineering, University of the West of Scotland, Paisley PA1 2BE, United Kingdom

${ }^{7}$ IFIC, CSIC-Universidad de Valencia, E-46071 Valencia, Spain

${ }^{8}$ Horia Hulubei National Institute of Physics and Nuclear Engineering, RO-77125 Bucharest-Magurele, Romania

${ }^{9} \mathrm{CEA}$, Centre de Saclay, IRFU/SPhN, F-91191 Gif-sur-Yvette, France

(Received 23 February 2011; published 25 July 2011)

\begin{abstract}
New $\gamma$ transitions have been identified in argon isotopes in ${ }^{40} \mathrm{Ar}+{ }^{208} \mathrm{~Pb}$ multiple transfer reactions by exploiting, in a fragment- $\gamma$ measurement, the new generation of magnetic spectrometers based on trajectory reconstruction coupled to large $\gamma$ arrays. The coupling of single-particle degrees of freedom to nuclear vibration quanta was discussed. The interpretation of the newly observed states within a particle-phonon coupling picture was used to consistently follow, via their excitation energies, the evolution of collectivity in odd Ar isotopes. The proposed level schemes are supported by the results of $s d$ - $p f$ shell-model calculations, which have been also employed to evaluate the strength functions of the populated states.
\end{abstract}

DOI: $10.1103 /$ PhysRevC.84.014325

PACS number(s): 25.70.Hi, 21.10.Re, 23.20.Lv, 29.30.Aj

\section{INTRODUCTION}

A significant amount of experimental data on heavy ion transfer reactions collected in the last decade have been shown to be quantitatively described in the reaction model which includes elementary degrees of freedom, surface vibrations, and single particles [1]. It is through the excitation of the elementary modes that energy and angular momentum are transferred from the relative motion to these intrinsic degrees of freedom and that mass and charge are exchanged among the two partners of the collision. This exchange leads to the population of moderately neutron-rich nuclei [2] in the region of the nuclear chart where challenging aspects in the behavior of the nuclear structure have been foreseen [3]. The revival of transfer reaction studies benefited from the construction of the new generation large solid-angle spectrometers based on trajectory reconstruction that reached an unprecedented efficiency and selectivity. The coupling of these spectrometers with large $\gamma$-ray arrays allowed the identification of individual transitions and excited states, their population pattern, and decay modes via particle- $\gamma$ coincidences (see, e.g., [4-10] and references therein).

The coupling of single-particle degrees of freedom to nuclear vibration quanta is essential for the description of many basic states in the vicinity of closed shells. The effects of such coupling are largely unexplored, in particular, whether and to what extent a population of states of particle-phonon nature is present in isotopic chains reached via multiple-particle transfer mechanism. In this work we address this question through studies of argon isotopes populated by the neutron pick-up reactions in ${ }^{40} \mathrm{Ar}+{ }^{208} \mathrm{~Pb}$. Results will be discussed within the $s d-p f$ large-scale shell-model calculations. The strength functions calculated in the shell-model framework will be used to infer on the structure of the strongly populated states.

\section{EXPERIMENT AND DATA ANALYSIS}

An ${ }^{40} \mathrm{Ar}$ beam was extracted by an ECR ion source and accelerated by means of the superconducting ALPI accelerator of the Laboratory Nazionali di Legnaro at $E_{\text {lab }}=255 \mathrm{MeV}$ onto a $300 \mu \mathrm{g} / \mathrm{cm}^{2}{ }^{208} \mathrm{~Pb}$ target. Projectile-like fragments have been identified with the large solid-angle magnetic spectrometer Prisma [11] placed in the vicinity of the grazing angle $\left(\theta_{\text {lab }}=54^{\circ}\right)$. We here briefly recall the Prisma detector system. A position-sensitive microchannel plate detector [12] is placed at the entrance of the spectrometer, providing a start signal for time-of-flight measurements and position signals. Ions pass through the optical elements of the spectrometer (a quadrupole and a dipole) and enter a focal plane [13] which is made of a parallel plate of multiwire type, providing timing and position signals with resolutions similar to the entrance detector. At the end of the focal plane is located an array of a transverse field multiparametric ionization chambers, providing nuclear charge (via $\Delta E$ ) and total energy $(E)$. The described detector system gives all the necessary information for the complete ion identification, which is performed via an event-by-event reconstruction of the trajectory inside the magnetic elements [14]. 


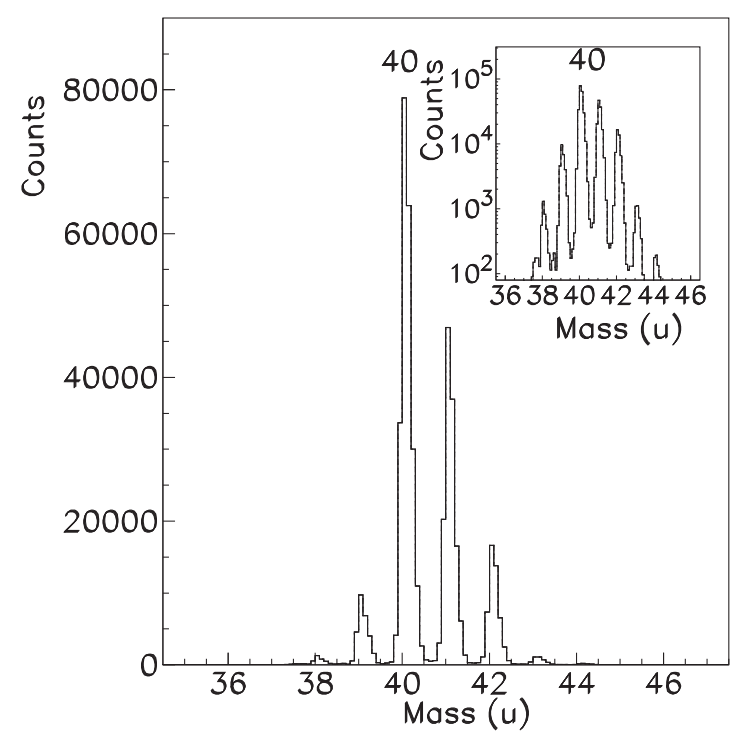

FIG. 1. Mass distribution of argon isotopes populated in ${ }^{40} \mathrm{Ar}+{ }^{208} \mathrm{~Pb}$ at $E_{\mathrm{lab}}=255 \mathrm{MeV}$ and at $\theta_{\mathrm{lab}}=54^{\circ}$ in coincidence with $\gamma$ rays. A mass resolution of $\Delta A / A \sim 1 / 160$ was obtained. The insert displays the same mass distribution in logarithmic scale.

To illustrate the achieved mass resolution the mass distribution of argon isotopes populated by neutron transfers was plotted in Fig. 1. One sees the dominance of neutron pick-up channels with a weaker population of neutron stripping channels, as expected from optimum $Q$-value arguments. In the neutron pick-up sector the yield drops almost by a constant factor by each transferred neutron as an independent particle mechanism would suggest [15]. The dependence of the yields on the number of transferred neutrons is very similar to the ones observed in other studied systems $[1,16,17]$. In the present case a deeper insight may be achieved by measuring the individual $\gamma$-ray transitions and thus to study how the relevant degrees of freedom acting in the reaction dynamics define the final single excited state distributions. We remind that the secondary fragments are detected and therefore the final isotopic distribution is affected by evaporation processes. These processes influence more significantly the neutron stripping channels $[16,17]$, thus, in the forthcoming analysis we focus on neutron pick-up channels.

The $\gamma$ rays were detected with the Clara array [18], which consists of $24 \mathrm{HP}-\mathrm{Ge}$ clover-type detectors situated in the hemisphere close to the target and opposite Prisma. The total photopeak efficiency is of the order of $3 \%$ for $E=1.33 \mathrm{MeV}$. The fragment $-\gamma$ coincidence obtained from the coupling of Clara with Prisma allows us to attribute to each specific reaction product its characteristic $\gamma$ rays. Doppler correction is obtained from the knowledge of the reconstructed velocity vector, with typical $\gamma$-ray energy resolutions of $0.6 \%-0.9 \%$ over the whole velocity distribution.

The $\gamma$ spectra of ${ }^{40,41,42,43} \mathrm{Ar}$ corresponding to the $0 n,+1 n$, $+2 n$ and $+3 n$ channels, respectively, have been plotted in Fig. 2. The strongest observed transitions and new transitions that have been identified in this work, $\left(9 / 2^{-} \rightarrow 7 / 2^{-}\right)$, $\left(11 / 2^{-} \rightarrow 7 / 2^{-}\right)$in ${ }^{41} \mathrm{Ar}$ and ${ }^{43} \mathrm{Ar}$, and $\left(6^{+} \rightarrow 4^{+}\right)$in ${ }^{42} \mathrm{Ar}$, will be discussed in more detail in the following sections.

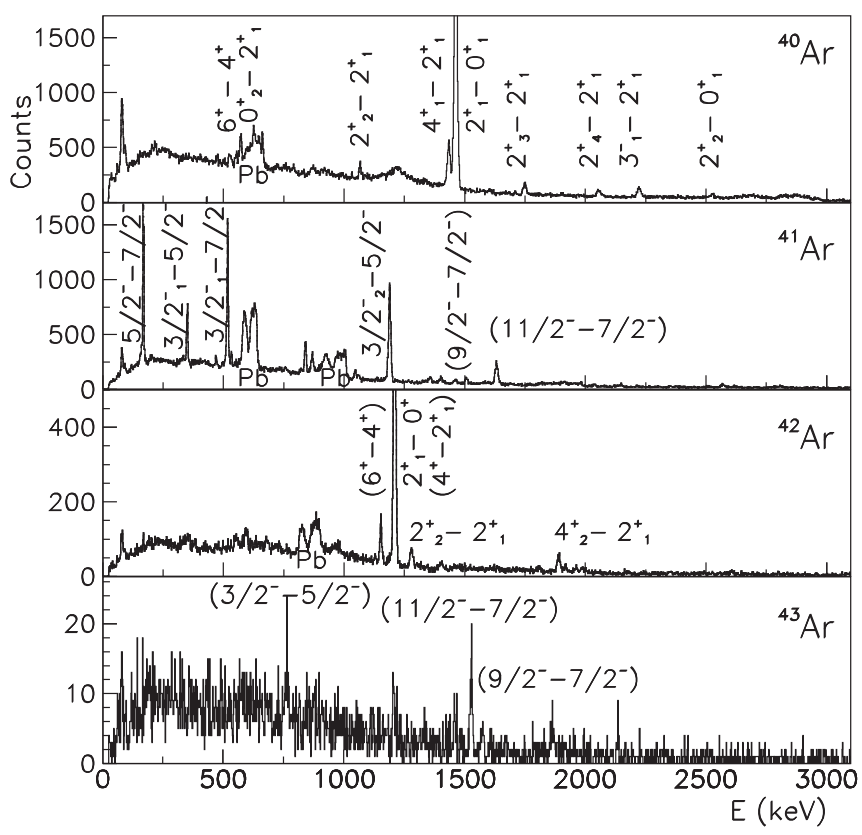

FIG. 2. Doppler corrected $\gamma$-ray spectra for ${ }^{40,41,42,43}$ Ar populated in ${ }^{40} \mathrm{Ar}+{ }^{208} \mathrm{~Pb}$ by detecting the $0 n,+1 n,+2 n$, and $+3 n$ channels in Prisma and the coincident $\gamma$ rays in Clara. The strongest transitions are marked in the figure, with the spin and parity as listed in Ref. [19]. New transitions that have been identified in this work, $\left(9 / 2^{-} \rightarrow\right.$ $\left.7 / 2^{-}\right),\left(11 / 2^{-} \rightarrow 7 / 2^{-}\right)$in ${ }^{41} \mathrm{Ar}$ and ${ }^{43} \mathrm{Ar}$, and $\left(6^{+} \rightarrow 4^{+}\right)$in ${ }^{42} \mathrm{Ar}$, are listed in more details in Table I, together with the here preferentially assigned spin $4^{+}$for the $2413-\mathrm{keV}$ state in ${ }^{42} \operatorname{Ar}\left[\left(3^{-}, 4^{+}\right)\right.$in Ref. [19]]. The label "Pb" refers to the wrongly Doppler corrected $\gamma$ rays of the heavy partners.

\section{POPULATED STATES AND SHELL-MODEL CALCULATIONS}

\section{A. Structure of even Ar isotopes}

In ${ }^{40} \mathrm{Ar}$ the strongest observed transitions are from $2^{+}, 4^{+}$, and $6^{+}$yrast states, with a very strong population of the $2^{+}$ state. In addition to them, we also observe a weaker feeding of the $3^{-}$state and of the nonyrast $2^{+}$and $0^{+}$states.

The strong line at $1208 \mathrm{keV}$ dominates the $+2 n$ channel. In addition to this line we also observe lines at 1150.4(3), 1277.5(3), and 1887.5(6) keV. From the adopted levels of ${ }^{42} \mathrm{Ar}$ [19] we recognize the 1278 - and $1888-\mathrm{keV}$ transitions as the nonyrast $2_{2}^{+} \rightarrow 2_{1}^{+}$and $4_{2}^{+} \rightarrow 2_{1}^{+}$transitions, respectively. Invoking the particle $-\gamma-\gamma$ coincidences depicted in Fig. 3 for the region around $1200 \mathrm{keV}$, we attributed the observed $\gamma$ rays to the de-excitation of the $2^{+}, 4^{+}$, and $6^{+}$yrast sequence, with excitation energies of 1208.4(6) $\mathrm{keV}, 2413(1) \mathrm{keV}$, and 3563(1) $\mathrm{keV}$, respectively. [In Ref. [19] the level at $2414.0(6) \mathrm{keV}$ is given as $\left(3^{-}, 4^{+}\right)$, where $(t, p)$ studies have preferences for $\left.4^{+}\right]$. The $\gamma$ rays of the transitions $2_{1}^{+} \rightarrow 0_{\text {g.s. }}^{+}$and $4_{1}^{+} \rightarrow 2_{1}^{+}$have very similar energies, which have been extracted from the particle $-\gamma-\gamma$ spectra as 1208(1) and $1205(1) \mathrm{keV}$, respectively. From the intensity of $\gamma$ lines and taking into account the $\gamma$ gated spectra, the 1150.4(3)-keV line was attributed to the $6_{1}^{+} \rightarrow 4_{1}^{+}$transition. More details about these states are presented in Table I. All these transitions, according to our present attribution of spin and parity, are 


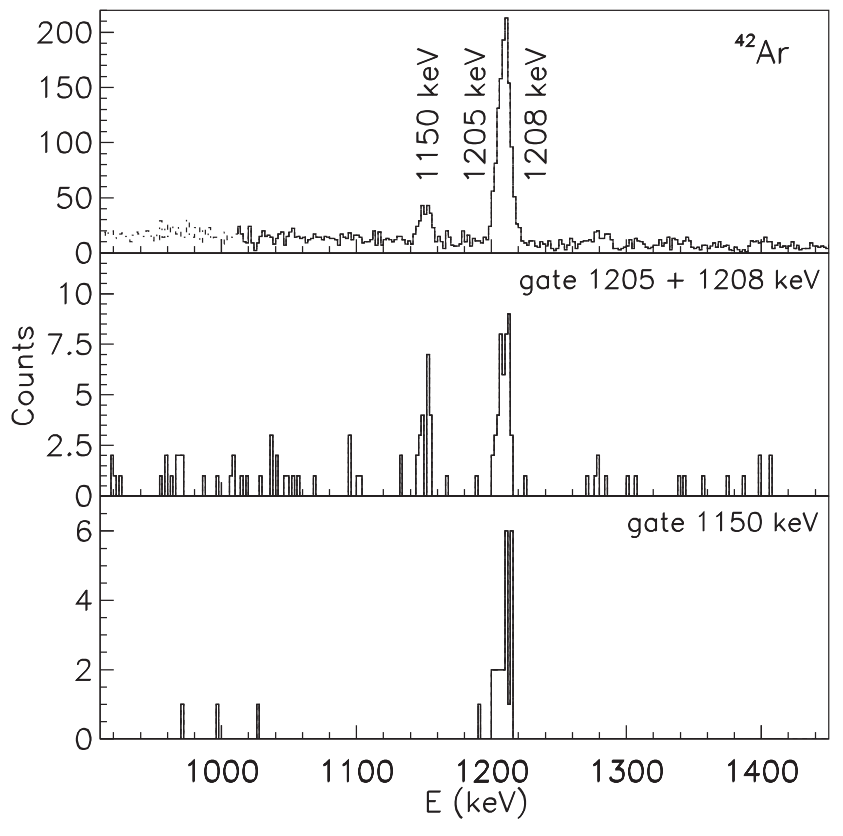

FIG. 3. $\gamma$-ray spectra in coincidence with ${ }^{42} \mathrm{Ar}$ zoomed in the range of interest (top panel), and with additional gates (particle$\gamma-\gamma$ coincidences) on $1205-1208-\mathrm{keV}$ (middle panel) or $1150-\mathrm{keV}$ (bottom panel) transitions. The 1208(1)- and 1205(1)-keV lines are in coincidence with each other, and at least one of them, probably both, are in coincidence with the 1150.4(3)-keV line.

depicted in Fig. 4 together with the $0 \hbar \omega s d$-pf large-scale shell-model (SM) calculations [20,21]. The recently developed SDPF-U effective interaction, with the valence space of the full $s d$ shell for the protons and the full $p f$ shell for the neutrons, was used. The results of SM calculations agree well with the experimental energy spectrum, both for the observed adopted transitions [19], and for the energies and spins of $4^{+}$and $6^{+}$ yrast states.

Looking at the spectra of the even argon isotopes we notice the strong population of the $2^{+}$state. From the reaction model we know that the excitation and transfer processes are mediated by the well-known single-particle form factors for the fermion degrees of freedom and by the collective form factors, for the vibrational modes. By comparison of the measured and SM calculated energy spectra we learned that the SM framework

TABLE I. New $\gamma$ transitions identified in the present work, and some of the known transitions or levels according to Ref. [19] to which additional information is added. The label $\Delta$ stands for the yet unknown $7 / 2^{-} \rightarrow 5 / 2^{-}$transition in ${ }^{43} \mathrm{Ar}$.

\begin{tabular}{lrrrrc}
\hline \hline Isotope & $E_{\gamma}(\mathrm{keV})$ & \multicolumn{1}{c}{$E_{i}(\mathrm{keV})$} & \multicolumn{1}{c}{$J_{i}^{\pi}$} & $E_{f}(\mathrm{keV})$ & $J_{f}^{\pi}$ \\
\hline${ }^{41} \mathrm{Ar}$ & $1629.7(3)$ & $1629.7(3)$ & $\left(11 / 2^{-}\right)$ & 0.0 & $7 / 2^{-}$ \\
& $1504.8(6)$ & $1504.8(6)$ & $\left(9 / 2^{-}\right)$ & 0.0 & $7 / 2^{-}$ \\
\multirow{2}{*}{$\mathrm{Ar}$} & $1150.4(3)$ & $3563(1)$ & $\left(6^{+}\right)$ & $2413(1)$ & $\left(4^{+}\right)$ \\
& $1205(1)$ & $2413(1)$ & $\left(4^{+}\right)$ & $1208(1)$ & $2^{+}$ \\
${ }^{43} \mathrm{Ar}$ & $762.3(4)$ & $762.3(4)$ & $\left(3 / 2^{-}\right)$ & 0.0 & $5 / 2^{-}$ \\
& $1527.4(5)$ & $1527.4(5)+\Delta$ & $\left(11 / 2^{-}\right)$ & $\Delta$ & $\left(7 / 2^{-}\right)$ \\
& $1859(2)$ & $1859(2)+\Delta$ & $\left(9 / 2^{-}\right)$ & $\Delta$ & $\left(7 / 2^{-}\right)$ \\
\hline \hline
\end{tabular}

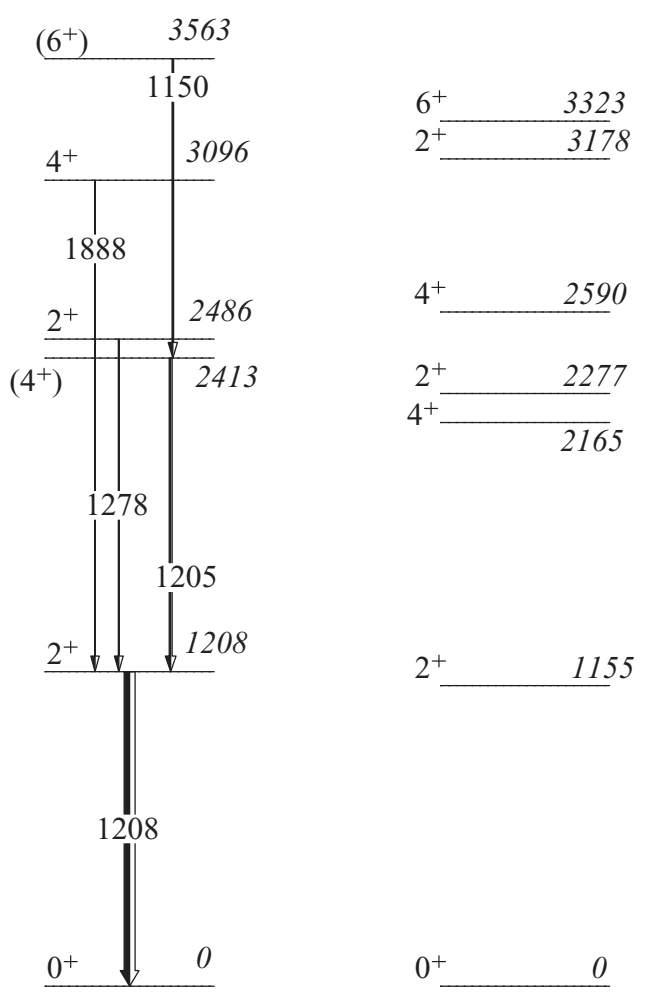

FIG. 4. The experimental (left) and SM calculated (right) level scheme of ${ }^{42} \mathrm{Ar}$, populated via $+2 n$ channel. Relative $\gamma$-ray intensities (left) are indicated by the width of the arrows. Only $\gamma$ transitions observed in our measurement are plotted. The energy, spin, and parity of levels, their branching ratios, and energy of transitions, where known, are as in Ref. [19]. We propose $\left(6^{+}\right)$for the new state at $3563 \mathrm{keV}$, and the spin $\left(4^{+}\right)$for the $2413-\mathrm{keV}$ state $\left[\left(3^{-}, 4^{+}\right)\right.$in Ref. [19]]. SM calculations for all positive parity states with the energy up to $3323 \mathrm{keV}$ are plotted (right).

describes well the observed energies, therefore we looked more closely at the SM calculated wave functions and the occupational probabilities for different orbitals, especially of the ground states and $2^{+}$states. These states, as will be demonstrated below, act as cores in the odd isotopes when a neutron is added. The overlap of their wave functions with the wave functions of the states in odd isotopes will be used in the construction of the strength functions (see Sec. IV). About $90 \%$ and $80 \%$ of squared amplitudes of these wave functions are with the two and four neutrons in $f_{7 / 2}$ for ${ }^{40} \mathrm{Ar}$ and ${ }^{42} \mathrm{Ar}$, respectively. The main component of the proton configuration is $\left(d_{5 / 2}\right)^{6}\left(s_{1 / 2}\right)^{2}\left(d_{3 / 2}\right)^{2}$; it amounts to $75 \%$ and $55 \%$ for the $0^{+}$state, and $71 \%$ and $49 \%$ for the $2^{+}$state in ${ }^{40} \mathrm{Ar}$ and ${ }^{42} \mathrm{Ar}$, respectively. With the addition of neutrons a single $s_{1 / 2}$ proton promotes to the $d_{3 / 2}$ orbital. When moving from ${ }^{40} \mathrm{Ar}$ to ${ }^{42} \mathrm{Ar}$ the occupation probability of $s_{1 / 2}$ decreases by $8 \%$. The growing importance of this proton configuration [i.e., $\left.\left(s_{1 / 2}\right)^{1}\left(d_{3 / 2}\right)^{3}\right]$ is related to the characteristic of the SDPF-U interaction which causes a narrowing of the gap between the $s_{1 / 2}$ and $d_{3 / 2}$ proton orbitals [21], when going toward more neutron-rich argon isotopes. In our SM calculations within $s d$ - $p f$ space, the depletion of $s_{1 / 2}$ strongly contributes to the increase of collectivity in the Ar isotopes with neutron number 


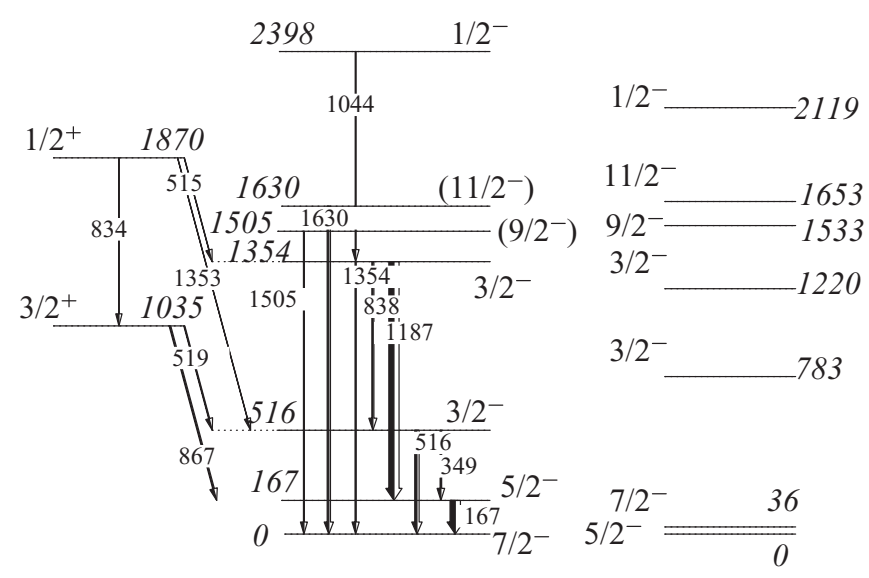

FIG. 5. The experimental and SM calculated level scheme of ${ }^{41} \mathrm{Ar}$ populated via the $+1 n$ channel for positive parity states (left), negative parity states (middle), and calculated (right) are plotted. Relative $\gamma$-ray intensities (left and middle) are indicated by the width of the arrows. Only $\gamma$ transitions observed in our measurement are plotted. The energy, spin, and parity of levels, the branching ratios, and the energy of transitions, when known, are as in Ref. [19]. For the new levels we propose the following assignments: $\left(9 / 2^{-}\right)$and $\left(11 / 2^{-}\right)$. SM calculations for all negative parity states with the energy up to $2119 \mathrm{keV}$ are plotted (right). Positive parity states could not be generated in the chosen $0 \hbar \omega$ valence space.

from 20 to 28 . In the following we will discuss in more details how this evolution could be followed also in the odd argon isotopes.

\section{B. Structure of odd Ar isotopes}

In ${ }^{41} \mathrm{Ar}$, one-neutron pick-up channel, the negative parity low-lying $3 / 2_{2}^{-}$state with a pronounced single-particle character, is strongly populated (see Figs. 2 and 5). In addition to the known $\gamma$ transitions of the low-lying states, we observe a strong line at $1629.7(3) \mathrm{keV}$, which we attribute to the population of the yet unknown $11 / 2^{-}$state. From particle- $\gamma-\gamma$ coincidences this line turns out not to be connected with other observed lines, thus it is understood as a transition to the ground state. A 1.64-MeV state was seen in the ${ }^{40} \operatorname{Ar}(\vec{d}, p)^{41} \mathrm{Ar}$ reaction [22] and attributed to a $3 / 2_{3}^{-}$state. However, this state was weakly excited and the $3 / 2^{-}$angular distribution was only qualitatively reproduced by the DWBA calculations [22]. In the present work SM calculations predict the $3 / 2_{3}^{-}$state at the much higher energy of $\sim 3 \mathrm{MeV}$.

The $7 / 2^{-}$ground state of ${ }^{41} \mathrm{Ar}$ is well described by a neutron in the $f_{7 / 2}$ orbital. By coupling this particle state with the $2^{+}$, one expects a multiplet of states $\left(3 / 2^{-}, \ldots, 11 / 2^{-}\right)$. The reaction mechanism does not populate the components of the multiplet uniformly but favors the high-spin stretched configuration. At low bombarding energies the transfer process maximizes the transferred angular momentum to allow a good matching between the orbital angular momentum of the involved states [23,24].

Thus we interpret the transition at 1629.7 (3) $\mathrm{keV}$ in ${ }^{41} \mathrm{Ar}$ as the decay from the $11 / 2^{-}$to the ground state. This agrees well with the SM calculation. On a similar basis, large angular
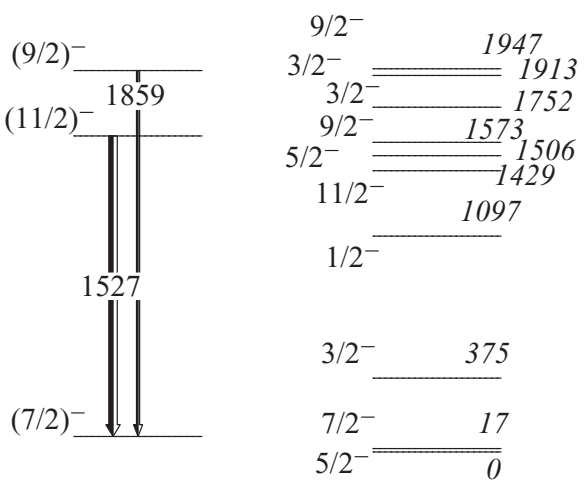

FIG. 6. The experimental and SM calculated level scheme of ${ }^{43} \mathrm{Ar}$, populated via $+3 n$ channel for negative parity states (left and middle) (being the absolute position of the $7 / 2^{-}$unknown, only relative position is indicated) and calculated in SM (right) are plotted. Relative $\gamma$-ray intensities (left and middle) are indicated by the width of the arrows. Only $\gamma$ transitions observed in our measurement are plotted. SM calculations for all negative parity states with the energy up to $2000 \mathrm{keV}$ are plotted (right).

momentum transfer and SM calculations, the new observed $\gamma$ transition of $1504.8(6) \mathrm{keV}$ is tentatively attributed to the $9 / 2^{-} \rightarrow 7 / 2^{-}$decay. The new experimental results for this isotope are also listed in Table I. A very good agreement between the experiment and SM calculated negative parity states, is reflected in a one-to-one correspondence between the calculated and observed levels.

Available information about spin and parity of ${ }^{43} \mathrm{Ar}$ is very scarce, coming solely from the $\beta^{-}$decay study [25] and $\left(p, p^{\prime}\right)$ reaction [26]. A recent measurement [27] performed via laser spectroscopy sets the spin of the ground state as $5 / 2^{-}$. In the present work several new transitions have been identified, which are depicted in Figs. 2 and 6. In odd Ar isotopes, with neutrons filling the $f_{7 / 2}-p_{3 / 2}$ shell, one sees a characteristic pattern of three low-energy $7 / 2^{-}, 5 / 2^{-}$, and $3 / 2^{-}$states. The here observed 762-keV transition was previously in Ref. [19] attributed to the decay of the $\left(3 / 2_{1}^{-}\right)$state to the $5 / 2^{-}$ground state.

The expected $7 / 2^{-}$state is still missing. Very recently, in ${ }^{9} \mathrm{Be}+{ }^{36} \mathrm{~S}$ fusion evaporation reaction a $\gamma$ ray of $201 \mathrm{keV}$ was reported [28] and attributed to the $7 / 2^{-} \rightarrow 5 / 2_{\text {g.s. }}^{-}$transition in ${ }^{43} \mathrm{Ar}$, as it could not be assigned to any other nucleus in the fusion channels. A 200-keV transition should have been observed in our particle- $\gamma$ measurement.

The (strong) line at $1527.4(5) \mathrm{keV}$ is a good candidate for the $11 / 2^{-}$to $7 / 2^{-}$transition. The proton scattering measurement [26] reported in ${ }^{43} \mathrm{Ar}$ an excited state at $1.61 \mathrm{MeV}$, which decays by a strong $E 2$ transition. The main conclusion of Ref. [26] is that this is a negative parity state that is dominated by a configuration where the valence neutrons occupy the $f p$ shell. Taking into account the main conclusion of Ref. [26] and the new results of Ref. [25], we attribute for this state a spin of $11 / 2$ decaying via a $1527-\mathrm{keV}$ transition. The missing $7 / 2^{-} \rightarrow 5 / 2_{\text {g.s. }}^{-}$transition should appear in a low-energy region, below $100 \mathrm{keV}$. This agrees well with the SM calculation, which predicts in ${ }^{43} \mathrm{Ar}$ an inversion of 
the two lowest states, $7 / 2^{-}$and $5 / 2^{-}$, which are almost degenerated, within $17 \mathrm{keV}$. Thus, we attribute the $1527 \mathrm{keV} \gamma$ ray to the $11 / 2^{-} \rightarrow 7 / 2^{-}$transition, with the $7 / 2^{-} \rightarrow 5 / 2_{\text {g.s. }}^{-}$ transition yet to be identified (labeled as $\Delta$ in Table I). Looking at the wave function a similar argument holds for the $9 / 2_{2}^{-}$state, which has a strong $2^{+} \otimes f_{7 / 2}$ component, very similar to $9 / 2_{1}^{-}$in ${ }^{41} \mathrm{Ar}$. Thus we tentatively attribute the here observed $1859(2)-\mathrm{keV} \gamma$ ray to the $9 / 2_{2}^{-} \rightarrow 7 / 2_{1}^{-}$ transition.

The low statistics for this channel did not allow one to establish coincidence relationships between the observed $\gamma$ lines, as was done for ${ }^{41} \mathrm{Ar}$ and ${ }^{42} \mathrm{Ar}$. Nevertheless, the close resemblance of the unknown energies with the attributed states in other odd $\mathrm{Ar}$ isotopes, and with the respective even-even cores suggest that the two transitions form the beginning of a structure built on the $7 / 2^{-}$state. The SM calculated and here proposed assignment for $11 / 2^{-}$agree well. As it turns out, the unobserved SM calculated negative parity states, have a weak single-particle contribution $\left(1 / 2_{1}^{-}\right.$and $\left.5 / 2_{2}^{-}\right)$, or their single-particle contribution is very fragmented $\left(3 / 2^{-}\right.$ states). These will be discussed in more detail in the next section.

\section{SINGLE-PARTICLE AND COLLECTIVE EXCITATIONS}

To better understand the structure of states in argon isotopes, we felt it would be interesting to calculate the strength functions of the odd isotopes in the framework of SM by using the Lanczos method and pivot states that corresponds to the creation of neutron in $v p_{1 / 2}, v p_{3 / 2}, v f_{7 / 2}$, and $v f_{5 / 2}$ on the $0^{+}$ground state of ${ }^{40} \mathrm{Ar}$ (left top panels in Fig. 7) and ${ }^{42} \mathrm{Ar}$ (right top panels in Fig. 7). They have been calculated through an iterative procedure, and using the overlap relation between the obtained eigenstates of the SM Hamiltonian and the pivot states $[20,29]$. The same procedure was applied to calculate the strength functions of a neutron in $v f_{7 / 2}$, coupled to $3 / 2^{-}, 5 / 2^{-}, 7 / 2^{-}, 9 / 2^{-}$, and $11 / 2^{-}$, on the $2^{+}$state of ${ }^{40} \mathrm{Ar}$ and ${ }^{42} \mathrm{Ar}$ (bottom panels in Fig. 7). Taking into account that the two configurations plotted in the top and bottom panels of Fig. 7 exhausted most of the strength of the low-lying $3 / 2^{-}$, $5 / 2^{-}$, and $7 / 2^{-}$states, we will explore in more detail what is the dominant component of their wave functions. It turns out that a large part of the $7 / 2_{1}^{-}$wave function in ${ }^{41} \mathrm{Ar}$ can be understood as $0^{+} \otimes f_{7 / 2}$, with a much weaker component of $2^{+} \otimes f_{7 / 2}$. In the case of ${ }^{43} \mathrm{Ar}$, the $7 / 2^{-}$state, displays a more equilibrated sharing between the $0^{+} \otimes v f_{7 / 2}$ and $2^{+} \otimes v f_{7 / 2}$ components. The overlap between the wave functions of $5 / 2_{1}^{-}$ and $2^{+} \otimes f_{7 / 2}$ is very large, whereas most of the strength of the $0^{+} \otimes f_{5 / 2}$ component is in $5 / 2^{-}$states with excitation energies of $\sim 5-6 \mathrm{MeV}$. The $5 / 2_{1}^{-}$state has a very weak single-particle contribution. The top panels of Fig. 7 clearly display a concentration of single-particle strength in $3 / 2_{2}^{-}$of ${ }^{41} \mathrm{Ar}$. The single-particle strength is more fragmented in ${ }^{43} \mathrm{Ar}$, leaving the $3 / 2_{1}^{-}$state as the one with the largest single-particle strength. The strength function of the $1 / 2^{-}$states shows that their single-particle strength is fragmented over several $1 / 2^{-}$ states.
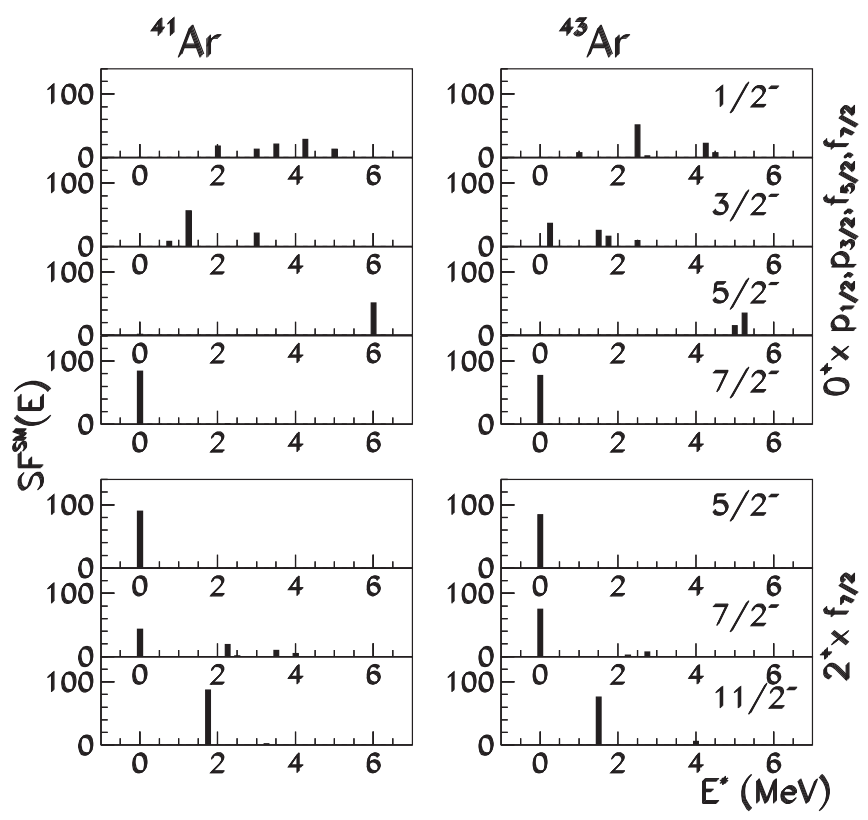

FIG. 7. The strength functions $\operatorname{SF}^{\mathrm{SM}}(E)$ calculated in the SM framework. They are displayed as percents of the strength function sum rule integrals in each panel. The top panels display the strength functions of the creation of a neutron in $p_{1 / 2}, p_{3 / 2}, f_{5 / 2}$, and $f_{7 / 2}$ on the $0^{+}$ground state of ${ }^{40} \mathrm{Ar}$ (left) and ${ }^{42} \mathrm{Ar}$ (right). The bottom panels show the strength functions of a neutron in $f_{7 / 2}$ coupled to the $2^{+}$ state of ${ }^{40} \mathrm{Ar}$ (left) and ${ }^{42} \mathrm{Ar}$ (right) for the states $5 / 2^{-}, 7 / 2^{-}$, and $11 / 2^{-}$

The bottom panels of Fig. 7 show a large concentration of $2^{+}$of ${ }^{40} \mathrm{Ar}$ and ${ }^{42} \mathrm{Ar}$ coupled to the neutron in $f_{7 / 2}$ in the $11 / 2^{-}$states. The $11 / 2_{1}^{-}$state, with a dominant $2^{+} \otimes v f_{7 / 2}$ stretched configuration, should decay to the $7 / 2^{-}$state of the dominant $0^{+} \otimes v f_{7 / 2}$ structure, with an $E 2$ strength similar to the one of the $2^{+} \rightarrow 0^{+}$transition in $A-1$ even isotopes. This is supported by the SM calculated $B(E 2)$ values; they turn out to be $48.65 e^{2} \mathrm{fm}^{4}$ and $70.27 e^{2} \mathrm{fm}^{4}$ for $2_{1}^{+} \rightarrow 0_{\text {g.s. }}^{+}$for ${ }^{40} \mathrm{Ar}$ and ${ }^{42} \mathrm{Ar}$, respectively, whereas for the $11 / 2_{1}^{-} \rightarrow 7 / 2_{1}^{-}$ in ${ }^{41} \mathrm{Ar}$ and ${ }^{43} \mathrm{Ar}$ they are $53.17 e^{2} \mathrm{fm}^{4}$ and $85.40 e^{2} \mathrm{fm}^{4}$, respectively. For $B(E 2)$ calculations the standard effective charges $e_{\pi}=1.5 e$ and $e_{v}=0.5 e$ have been used. Of course, SM predictions of $E 2$ strength need to be validated by experiments.

Here we make an attempt to understand the feeding of the states populated via transfer mechanism from the study of experimental yields. In Table II are reported the intensities for different states in ${ }^{41} \mathrm{Ar}$ and ${ }^{43} \mathrm{Ar}$. Those for ${ }^{41} \mathrm{Ar}$ have been obtained by subtraction of the feeding from above in agreement with the level scheme of Fig. 5. Those for ${ }^{43} \mathrm{Ar}$, because of poorer statistics (see Fig. 6), are just the measured intensities. The intensity of each transition was normalized to the strongest transition in each isotope, and corrected for the efficiency of the $\gamma$ array. From Table II we see that the states with a pronounced single-particle character are strongly excited by the transfer mechanism (i.e., the $3 / 2_{2}^{-}$state in ${ }^{41} \mathrm{Ar}$ and the $3 / 2_{1}^{-}$state in ${ }^{43} \mathrm{Ar}$ ). On the other hand, the $5 / 2-$ state in ${ }^{41} \mathrm{Ar}$, which has a small single-particle component, turns out to have a negligible 
TABLE II. Experimental intensities of the populated excited states, $I^{\mathrm{EXP}}$, for ${ }^{41} \mathrm{Ar}$ and ${ }^{43} \mathrm{Ar}$. In the case of ${ }^{41} \mathrm{Ar}$ the intensities have been obtained by subtractions of the feeding from above in agreement with the level scheme presented in Fig. 5. In the case of ${ }^{43} \mathrm{Ar}$, according to the level scheme presented in Fig. 6, no subtraction was applied. The intensity of each transition was normalized to the strongest transition in ${ }^{41} \mathrm{Ar}$ and ${ }^{43} \mathrm{Ar}$, and corrected for the efficiency of the $\gamma$ array. Quoted errors take into account the statistic $\left({ }^{41} \mathrm{Ar}\right.$ and $\left.{ }^{43} \mathrm{Ar}\right)$ and subtraction procedure $\left({ }^{41} \mathrm{Ar}\right)$. The label $\Delta$ stands for the yet unknown $7 / 2^{-} \rightarrow 5 / 2^{-}$transition in ${ }^{43} \mathrm{Ar}$.

\begin{tabular}{lccc}
\hline \hline Isotope & $J_{i}^{\pi}$ & $\begin{array}{c}E \\
(\mathrm{keV})\end{array}$ & $\begin{array}{c}I^{\mathrm{EXP}} \\
(\%)\end{array}$ \\
\hline${ }^{41} \mathrm{Ar}$ & $1 / 2^{-}$ & 2398 & $7(1)$ \\
& $3 / 2_{1}^{-}$ & 516 & $30(6)$ \\
& $3 / 2^{-}$ & 1354 & $100(3)$ \\
& $\left(9 / 2^{-}\right)$ & 1505 & $8(1)$ \\
& $\left(11 / 2^{-}\right)$ & 1630 & $27(2)$ \\
${ }^{43} \mathrm{Ar}$ & $3 / 2^{-}$ & 763 & $64(21)$ \\
& $\left(9 / 2_{2}^{-}\right)$ & $1859+\Delta$ & $37(15)$ \\
& $\left(11 / 2^{-}\right)$ & $1527+\Delta$ & $100(16)$ \\
\hline \hline
\end{tabular}

direct feeding at least within the relatively large errors of such a procedure. We here remind that in this fragment $\gamma$ coincidence measurement we cannot extract the ground-state population, but from $Q$-value matching conditions it is foreseen to be strong.

In addition to the states of single-particle character, we also see from Table II that for ${ }^{41} \mathrm{Ar}$ we populate significantly the $11 / 2_{1}^{-}$state, with about one-third of the strength of the strongest populated single-particle state. As discussed before, for this state the shell-model strength function calculations point to a large concentration of $2^{+}$of ${ }^{40} \mathrm{Ar}$ coupled to the neutron in $f_{7 / 2}$. On a similar basis we interpreted the $1527-\mathrm{keV}$ $\gamma$ in ${ }^{43} \mathrm{Ar}$ as the $E 2$ transition from the strongly populated $11 / 2^{-}$state. The population of these states is not unexpected because the importance of the nuclear vibration quanta and its coupling to the single-particle degrees of freedom was recognized in the reaction model [30].

The properties of the particle-phonon states should be to a large extent determined by the properties of the corresponding phonon states. The comparison of the measured and SM calculated energies for the $2^{+}$and $11 / 2^{-}$states of argon isotopes in the range $N=20-28$ is shown in Fig. 8 . An excellent agreement for all argon isotopes between the experiment and SM is obtained. The figure clearly displays that the evolution of the collectivity, in the even isotopes $\left(2^{+}\right.$ energies) and in the odd isotopes $\left(11 / 2^{-}\right.$energies), is very similar. This further corroborates the particle-phonon character of these $11 / 2^{-}$states. Heavy-ion-induced transfer reactions should populate states of similar character in more neutron-rich isotopes. Argon isotopes with $N \geqslant 28$ have been populated in ${ }^{238} \mathrm{U}+{ }^{48} \mathrm{Ca}$ reactions [34]. The observed transitions in ${ }^{47} \mathrm{Ar}$, with $3 / 2^{-}$as a ground state, have been associated with the low-lying $\left(1 / 2^{-}\right),\left(5 / 2^{-}\right)$, and $\left(7 / 2^{-}\right)$states, where the $7 / 2^{-}$ state was seen as the coupling of a $p_{3 / 2}$ neutron to $2^{+}$of ${ }^{46} \mathrm{Ar}$. This situation is similar to ${ }^{41} \mathrm{Ar}$ and ${ }^{43} \mathrm{Ar}$ discussed here, where the strongly populated $11 / 2^{-}$states have been

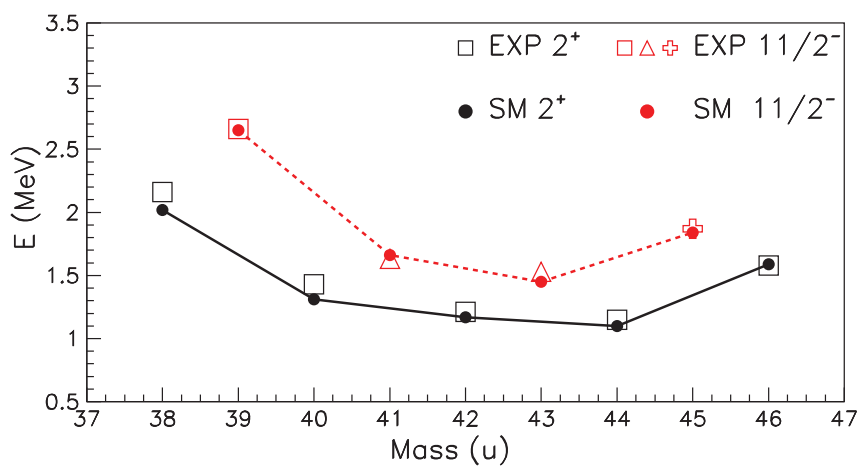

FIG. 8. (Color online) Energies of the $2^{+}$and $11 / 2^{-}$states of argon isotopes with $N=20-28$. Solid circles are SM calculated energies, open squares are the adopted levels, whereas open triangles correspond to the energies of $11 / 2^{-}$in ${ }^{41} \mathrm{Ar}$ and ${ }^{43} \mathrm{Ar}$ from the present work. In the most recent compilation of the ${ }^{45} \mathrm{Ar}$ level properties [31], a level at $1911(5) \mathrm{keV}$ is reported to decay by $100 \%$ to the $7 / 2^{-}$ ground state. This level is a good candidate for the $11 / 2^{-}$state in this nucleus [32,33], and was added (cross symbol) for completeness. Curves are here only to guide eyes.

described as the $f_{7 / 2}$ neutron coupled to the $2^{+}$states of ${ }^{40} \mathrm{Ar}$ and ${ }^{42} \mathrm{Ar}$.

\section{SUMMARY}

Multinucleon transfer reactions have been investigated in ${ }^{40} \mathrm{Ar}+{ }^{208} \mathrm{~Pb}$ via particle- $\gamma$ coincidences with the Prisma+Clara setup. New states have been identified and the strongly populated states have been discussed and compared with SM calculations. In odd Ar isotopes, we reported, in addition to the excitation of the states of single-particle character, a significant population of proposed $11 / 2^{-}$states, reached via neutron transfer. The structure of these $11 / 2^{-}$states matches a stretched configuration of the valence neutron coupled to the vibration quanta. The properties of such states are closely connected with the properties of the vibration quanta, allowing one to follow the development of collectivity in odd argon isotopes, a phenomenon widely discussed in even-even isotopes. The individual state yield distribution in the final reaction products reflects a strong interplay between single-particle and collective degrees of freedom and the reaction dynamics. These effects are expected to be important also for very neutron-rich nuclei and the research using radioactive beams as a probe.

\section{ACKNOWLEDGMENTS}

The authors are grateful to the LNL ALPI staff for providing us with the good-quality beams and the target laboratory for the excellent target preparation. This work was partly supported by the European Community FP6- Structuring the ERA-Integrated Infrastructure Initiative (EURONS Contract No. RII3-CT-2004-506065), and by the "Ministère des Affaires étrangères et européennes" in the Partnership Hubert Curien, program (PHC) Cogito framework. This work was also supported in part by the Croatian Ministry of Science, Education and Sports (Grant No. 0098-1191005-2890). 
[1] L. Corradi, G. Pollarolo, and S. Szilner, J. Phys. G 36, 113101 (2009), and references therein.

[2] R. Broda, J. Phys. G 32, R151 (2006), and references therein.

[3] O. Sorlin and M.-G. Porquet, Prog. Part. Nucl. Phys. 61, 602 (2008), and references therein.

[4] N. Marginean et al., Phys. Lett. B 633, 696 (2006).

[5] S. Lunardi et al., Phys. Rev. C 76, 034303 (2007).

[6] B. Fornal et al., Phys. Rev. C 77, 014304 (2008).

[7] J. J. Valiente-Dobon et al., Phys. Rev. Lett. 102, 242502 (2009).

[8] S. Bhattacharyya et al., Phys. Rev. C 79, 014313 (2009).

[9] Z. M. Wang et al., Phys. Rev. C 81, 054305 (2010).

[10] J. Ljungvall et al., Phys. Rev. C 81, 061301 (2010).

[11] A. M. Stefanini et al., Nucl. Phys. A 701, 217c (2002).

[12] G. Montagnoli et al., Nucl. Instrum. Methods Phys. Res., Sect. A 547, 455 (2005).

[13] S. Beghini et al., Nucl. Instrum. Methods Phys. Res., Sect. A 551, 364 (2005).

[14] S. Szilner et al., Phys. Rev. C 76, 024604 (2007).

[15] A. Winther, Nucl. Phys. A 572, 191 (1994); 594, 203 (1995).

[16] L. Corradi, A. M. Stefanini, C. J. Lin, S. Beghini, G. Montagnoli, F. Scarlassara, G. Pollarolo, and A. Winther, Phys. Rev. C 59, 261 (1999).

[17] S. Szilner et al., Phys. Rev. C 71, 044610 (2005).

[18] A. Gadea et al., Eur. Phys. J. A 20, 193 (2004).
[19] J. A. Cameron and B. Singh, Nucl. Data Sheets 92, 1 (2001); 92, 783 (2001); 94, 429 (2001); National Nuclear Data Center [http://www.nndc.bnl.gov].

[20] E. Caurier, G. Martínez-Pinedo, F. Nowacki, A. Poves, and A. P. Zuker, Rev. Mod. Phys. 77, 427 (2005).

[21] F. Nowacki and A. Poves, Phys. Rev. C 79, 014310 (2009).

[22] S. Sen, S. E. Darden, W. A. Yoh, and E. D. Berners, Nucl. Phys. A 250, 45 (1975).

[23] A. R. Barnett, W. R. Phillips, P. J. A. Buttle, and L. J. B. Goldfarb, Nucl. Phys. A 176, 321 (1971).

[24] D. M. Brink, Phys. Lett. B 40, 37 (1972).

[25] J. A. Winger, P. F. Mantica, and R. M. Ronningen, Phys. Rev. C 73, 044318 (2006).

[26] F. Marechal et al., Phys. Rev. C 60, 064623 (1999).

[27] K. Blaum, W. Geithner, J. Lassen, P. Lievens, K. Marinova, and R. Neugart, Nucl. Phys. A 799, 30 (2008).

[28] M. Moralles, P. Janker, K. E. G. Lobner, and P. R. Pascholati, Phys. Rev. C 79, 037302 (2009).

[29] R. R. Whitehead, in Moment Methods in Many Fermion Systems, edited by D. J. Dalton, S. M. Grimes, J. D. Vary, and S. A. Williams (Plenum, New York, 1980), p. 235.

[30] R. A. Broglia and A. Winther, Heavy Ion Reactions (AddisonWesley, Redwood City, 1991).

[31] T. W. Burrows, Nucl. Data Sheets 109, 171 (2008).

[32] Zs. Dombrádi et al., Nucl. Phys. A 727, 195 (2003).

[33] D. Mengoni et al., Phys. Rev. C 82, 024308 (2010).

[34] S. Bhattacharyya et al., Phys. Rev. Lett. 101, 032501 (2008). 